\title{
A New Approach for the Characterization of Nonstationary Oscillators Using the Wigner-Ville Distribution
}

\author{
Chagai Levy $\left(\mathbb{D}\right.$, Monika Pinchas $\left(\mathbb{D}\right.$, and Yosef Pinhasi $\mathbb{i}^{\mathrm{D}}$ \\ Department of Electrical and Electronic Engineering, Ariel University, Ariel 40700, Israel \\ Correspondence should be addressed to Chagai Levy; chagailevy@gmail.com
}

Received 28 February 2018; Revised 9 June 2018; Accepted 20 June 2018; Published 11 July 2018

Academic Editor: Akhil Garg

Copyright (C) 2018 Chagai Levy et al. This is an open access article distributed under the Creative Commons Attribution License, which permits unrestricted use, distribution, and reproduction in any medium, provided the original work is properly cited.

\begin{abstract}
Oscillators and clocks are affected by physical mechanisms causing amplitude fluctuations, phase noise, and frequency instabilities. The physical properties of the elements composing the oscillator as well as external environmental conditions play a role in the characteristics of the oscillatory signal produced by the device. Such instabilities demonstrate frequency drifts and modulation and spectrum broadening and are observed to be nonstationary processes in nature. Most of tools which are being used to measure and characterize oscillator stability are based on signal processing techniques, assuming time invariance during a temporal window, during which the signal is assumed to be stationary. This paper proposes a new time-frequency metric for the characterization of frequency sources. Our technique is based on the Wigner-Ville distribution, which extends the spectral measures to consist of the temporal nonstationary behavior of the processes affecting the accuracy of the clock. We demonstrate the use of the technique in the characterization of phase errors, frequency offsets, and frequency drift of oscillators.
\end{abstract}

\section{Introduction}

In recent years, due to the high data rates required to be transferred in communication networks, the demand for high accuracy satellite navigation systems and the development of high resolution radars and clocks with very high precision are required. Stable oscillators, including atomic ones like rubidium and even cesium atomic, are often used in communication and navigation facilities [1-6]. In addition to the above applications, many other electronic systems require frequency generators. In such systems, time synchronization and an accurate clock frequency are required [7-12].

Every oscillator has its unique clock frequency consisting of a nominal frequency $v_{0}$ in addition to a normalized frequency difference of $x$ Parts Per Million (PPM) from the nominal frequency multiplied by this nominal frequency. In addition, every oscillator has a random jitter $\phi$. Therefore, the master and the slave clocks may have different frequencies and thus not be synchronized. For example, suppose that the master's frequency is $\left(v_{0}+x v_{0}\right)$ and the slave's frequency is $\left(v_{0}-x v_{0}\right)$; thus, their frequencies are not synchronized. In that case, the clock offset generally keeps increasing and may cause the communication link to fail [13]. Therefore, in such systems, frequency synchronization and an accurate clock frequency are required [14].

For example [13], communication networks such as Long Term Evolution Advanced (LTE-A) based cellular networks require base stations and backhaul equipment to maintain time synchronization in order to provide several new features like synchronized transmissions over frequencies from adjacent base stations and interference coordination. An example of an emerging networked application is smart power grid systems which are characterized by a two-way flow of energy and end-to-end communications. The communications are largely machine-to-machine (M2M) in nature $[13,15]$ and need to share synchronized information in order to improve efficiency and reliability of power delivery. Therefore, in addition to frequency synchronization, time synchronization is also required [16].

Oscillators and clocks are subjected to environmental conditions, internal malfunctions, and inherent physical phenomena causing instabilities in the oscillatory signal being produced [17]. These instabilities are expressed by amplitude and phase fluctuations causing frequency deviations, drift, and spectrum broadening. Instead of being stationary, single frequency, and temporal coherent, the clock signal is 
observed to be a stochastic process characterized by statistical and spectral features, which are nonstationary time varying [18-21]. Therefore, the design of a set of tools for time domain analysis of a frequency source is therefore needed and significant to investigate.

Several works [22-25] on the time-frequency (TF) analysis of oscillators and atomic clocks have been carried out recently. In $[22,23]$, the authors presented methods for the detection and identification of atomic clock anomalies via simulation on experimental data. Those methods are based on derivation of the frequency deviation and then sliding Welchs periodogram on the clock noise data.

Another work [24] presents a time-frequency relationship between the Langevin equation and the harmonic oscillator by deriving relationship between the Wigner distribution of the Green's function of the Langevin equation and of the harmonic oscillator. Their result paves the way for a simplification of the time-frequency representation of differential equations, as well as for a better understanding and filtering of the interference terms.

Most recently, a study of the important role of timefrequency signal representations was presented [25]. This approach was used for enhancement of the performance of global navigation satellite system (GNSS) receivers by using short-time Fourier transform (STFT) and the Wigner distribution as the TF representations.

In order to measure and characterize the oscillator properties, time-frequency signal processing techniques are required. Most often the Allan variance (or the Allan deviation) is employed [26-32]. It calculates the covariance of two adjacent samples of the signal produced by the under test oscillator. The Allan variance is widely used and recommended in international standards [28]. However, the Allan variance does not give the possibility of identifying and interpreting nonstationary effects.

Recently [18-21], an extended version of the Allan variance, the dynamic Allan variance (DAVAR), was proposed for the characterization of atomic clock stability that can vary with time. However, the DAVAR deals only with characterization of the stability of the frequency source and cannot detect phase and frequency offsets relative to a reference source.

The Allan variance is a fundamental tool for characterizing frequency stability of oscillators since it measures the size of frequency fluctuations at different observation intervals, whereas the DAVAR describes the variations with time of these fluctuations. Please note that in this paper we concentrate in ideal oscillators, where no random frequency fluctuations are considered.

In this paper we propose a time-frequency metric for the characterization of oscillators and frequency generators, considering also nonstationary variations. It is based on the Wigner distribution [33, 34], proposed in 1932 for the characterization of quantum fluctuations. In signal processing, it was used by Ville [35], so that it is often called Wigner-Ville distribution [36]. The applications of Wigner distribution are various: analysis of nonstationary signals [37-40], radar signals [41, 42], biomedical signals [43-45], analysis of time varying filters $[46,47]$, and image processing [48-50]. However, the Wigner-Ville distribution was not yet used in the characterization of nonstationary oscillators such as detection of the phase and frequency offsets relative to a reference source. In this paper, we will introduce both the discrete and continuous forms of the Wigner-Ville distribution for the characterization of a clock signal (nonstationary oscillator) with respect to a reference source and show how this technique is useful for the detection of the phase and frequency offsets relative to a reference source.

A short review of the oscillator clock model and the Wigner-Ville distribution are introduced in Section 2. The proposed approach for the characterization of nonstationary oscillators is presented in Sections 3-6. In Section 7 numerical simulation results are presented including demonstration of phase and frequency deviations. Section 8 summarizes the paper.

\section{Theoretical Background}

In this section, we will introduce a short theoretical background of the clock model and the Wigner-Ville distribution. In Section 2.1 we will describe briefly the clock model and in Section 2.2 we will present briefly the Wigner-Ville distribution [33].

2.1. The Clock Model. A model for a real clock which includes variations in the amplitude and phase is given by $[17,26]$

$$
r(t)=[A+\epsilon(t)] \cos \left[2 \pi v_{0} t+\varphi(t)\right]
$$

where $A$ is the amplitude and $\nu_{0}$ is the frequency of the oscillation. $\epsilon(t)$ and $\varphi(t)$ represent random fluctuations observed in amplitude and phase, respectively.

The amplitude fluctuations can be neglected [17, 26], and thus we can write the approximate model [17]:

$$
r(t) \simeq A \cos \left[2 \pi \nu_{0} t+\varphi(t)\right]
$$

The phase variations $\varphi(t)$ result in an instantaneous frequency which is a time dependent process given by [17]

$$
\nu(t)=v_{0}+\frac{1}{2 \pi} \frac{d \varphi(t)}{d t}
$$

In an ideal clock, the phase is constant and can be set to zero [51]; thus, it generates a sinusoidal oscillation of the form [52, 53]:

$$
s(t)=A_{0} \cos \left(2 \pi v_{0} t\right)
$$

where $A_{0}$ is the amplitude of the ideal clock.

Physical signals are real, in which case the Wigner-Ville distribution can be applied to either the (real) signal itself, or a complex version of the signal known as the analytic signal [54].

In the following, we define the analytic form of the clock signal as

$$
\tilde{r}(t) \simeq A e^{i\left[2 \pi v_{0} t+\varphi(t)\right]}
$$

and for an ideal clock, the analytic signal will be

$$
\widetilde{\boldsymbol{s}}(t)=A_{0} e^{i\left(2 \pi v_{0} t\right)}
$$


2.2. The Wigner-Ville Distribution. One of the most representative joint representations of time-frequency analysis is the Wigner distribution (WD), which is a quadratic signal representation introduced by Wigner (1932) [34] and later applied by Ville (1948) [35] to signal analysis. A comprehensive discussion of the WD properties can be found in a series of classical articles by Claasen and Mecklenbruker (1980) [55-57]. Originally, the WD has been applied to continuous variables as follows: consider an arbitrary $1 \mathrm{D}$ function $\widetilde{r}(t) \epsilon$ $\mathbb{C}, t \in \mathbb{R}$. The WD of $\widetilde{r}(t)$ is given by [33]

$$
W_{\tilde{r}}(t, f)=\int_{-\infty}^{\infty} \tilde{r}\left(t+\frac{\tau}{2}\right) \tilde{r}^{*}\left(t-\frac{\tau}{2}\right) e^{-i 2 \pi f \tau} d \tau
$$

where “*” denotes complex conjugation. By considering the shifting parameter $\tau$ as a variable, (7) represents the Fourier transform (FT) of the product $\tilde{r}(t+\tau / 2) \tilde{r}^{*}(t-\tau / 2)$, where $f$ denotes the spatial frequency variable and, hence, the WD can be interpreted as the local spectrum of the signal $\widetilde{r}(t)$.

The Wigner distribution satisfies many meaningful mathematical properties that are summarized in Appendix A.

The most powerful property is the "Time-Marginal" property and is given by

$$
\int_{-\infty}^{\infty} W_{\widetilde{r}}(t, f) d f=\|\widetilde{r}(t)\|^{2}
$$

where $\|\widetilde{r}(t)\|$ denotes the norm of the signal $\tilde{r}(t)$. Summing up of the energy distribution for all frequencies at a particular time would give the instantaneous energy of the signal. We will use the "Time-Marginal" property in our development in order to detect phase offset of a frequency source with respect to a reference source.

Modern signal processing uses the discrete form of physical signals. Therefore, the practical computation will use the discrete Wigner-Ville distribution form. There are several presentations in the literature for the discrete WignerVille distribution. In Appendix B we present and explain in detail the discrete form of the Wigner-Ville Distribution and some of the main presentations. In this paper we will use the following discrete Wigner-Ville distribution, similar to the one proposed by Eric Chassande-Mottin and Archana Pai [58]:

$$
w_{\tilde{r}}(n, m)=t_{s} \sum_{k=-k_{n}}^{k_{n}} \tilde{r}\left[n+\frac{k}{2}\right] \widetilde{r}^{*}\left[n-\frac{k}{2}\right] e^{-i 2 \pi m k /(2 N)}
$$

where $k_{n}=\min [2 n, 2 N-1-2 n], N$ denotes the number of samples, and $n, m$ denotes the index numbers for the time and frequency vectors, respectively.

This equation, designed for discrete time or space signals, shows a great similarity with the original continuous version; therefore, it has been selected for our development.

\section{A New Metric for Frequency Source Characterization}

The system under consideration is presented in Figure 1.

At first we will derive a general expression for $W_{\widetilde{r}-\widetilde{s}}(t, f)$ as a function of $\varphi(t)$; then we will develop simplified and

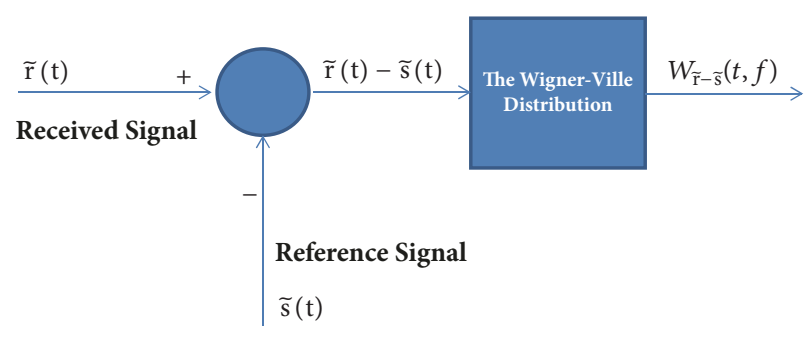

FIGURE 1: Block diagram of the proposed system. First we subtract the reference signal $\widetilde{\boldsymbol{s}}(t)$ from the received one $\widetilde{r}(t)$, and then the Wigner-Ville distribution function is performed on the difference $\widetilde{r}(t)-\widetilde{s}(t)$.

closed expression for the Time Marginal of $W_{\widetilde{r}-\widetilde{s}}(t, f)$ for three different cases.

Case A. In Section 4, we derive the analytic continuous and discrete forms for the Wigner-Ville distribution and its TimeMarginal expression for a constant phase offset case.

Case B. In Section 5, we derive the analytic expressions for the continuous Wigner-Ville distribution and its TimeMarginal expression for the case of constant phase and frequency offsets.

Case C. In Section 6, we derive the analytic expressions for the continuous Wigner-Ville distribution and its TimeMarginal expression for the case of frequency drift.

In this paper, we make use of an analytic signal which is the complex form of the real clock signal. Our choice is based on [54] because of the following reasons. All of the transformations in Cohens class of distributions (of which the Wigner-Ville is one) produce better results when applied to a modified version of the waveform termed the analytic signal, a complex version of the real signal. While the real signal can be used, the analytic signal has several advantages. The most important advantage is due to the fact that the analytic signal does not contain negative frequencies, so its use will reduce the number of cross products. If the real signal is used, then both the positive and negative spectral terms produce cross products.

Theorem 1. For the following assumptions:

(1) without loss of generality, $A=A_{0}=1$,

(2) $v_{0}$ is the nominal frequency,

(3) $\widetilde{s}(t)$ denotes the continuous complex form of an ideal clock signal,

(4) $\tilde{r}(t)$ denotes the continuous complex form of the real clock signal.

The Wigner-Ville distribution of the difference $\widetilde{r}(t)-\widetilde{s}(t)$ is defined as

$$
\begin{aligned}
W_{\tilde{r}-\tilde{s}}(t, f)=\delta & \left(f-v_{0}\right) \\
& +\int_{-\infty}^{\infty} e^{i \varphi(t+\tau / 2)} e^{-i \varphi(t-\tau / 2)} e^{-i 2 \pi\left(f-\nu_{0}\right) \tau} d \tau \\
& -2 \operatorname{Re}\left\{\int_{-\infty}^{\infty} e^{i\left[\left(2 \pi v_{0} \tau\right)+\varphi(t+\tau / 2)\right]} e^{-i 2 \pi f \tau} d \tau\right\}
\end{aligned}
$$


Proof of Theorem 1. The Wigner-Ville distribution (WD) of $\tilde{r}-\tilde{s}$ is given by

$$
W_{\widetilde{r}-\widetilde{s}}(t, f)=W D\left\{e^{i\left[2 \pi v_{0} t+\varphi(t)\right]}-e^{i\left(2 \pi v_{0} t\right)}\right\}
$$

By using the "Summation Property" given in Appendix A (A.2) we obtain

$$
\begin{aligned}
W_{\tilde{r}-\widetilde{s}}(t, f)= & W_{\widetilde{r}}(t, f)+W_{-\widetilde{s}}(t, f) \\
& -2 \operatorname{Re}\left\{W_{\widetilde{r}, \widetilde{s}}(t, f)\right\}
\end{aligned}
$$

Note that $W_{-\widetilde{s}}(t, f)=W_{\widetilde{s}}(t, f)$ for $\widetilde{s}(t) \in \mathbb{C}$. Then, by using (12) we have

$$
W_{\widetilde{r}-\widetilde{s}}(t, f)=W_{\widetilde{r}}(t, f)+W_{\widetilde{s}}(t, f)-2 \operatorname{Re}\left\{W_{\widetilde{r}, \widetilde{s}}(t, f)\right\}
$$

Let us get first a closed form expression for the second component in the right side of (13)

$$
\begin{aligned}
W_{\widetilde{s}}(t, f) & =\int_{-\infty}^{\infty} \widetilde{s}\left(t+\frac{\tau}{2}\right) \widetilde{s}^{*}\left(t-\frac{\tau}{2}\right) e^{-i 2 \pi f \tau} d \tau \\
& =\int_{-\infty}^{\infty} e^{i\left[2 \pi v_{0}(t+\tau / 2)\right]} e^{-i\left[2 \pi v_{0}(t-\tau / 2)\right]} e^{-i 2 \pi f \tau} d \tau \\
& =\int_{-\infty}^{\infty} e^{-i 2 \pi\left(f-\nu_{0}\right) \tau} d \tau=\delta\left(f-v_{0}\right)
\end{aligned}
$$

In order to get a closed form expression for the first component on the right side of (13) we will use the next definition:

$$
\widetilde{r}(t)=e^{i\left[2 \pi v_{0} t+\varphi(t)\right]}=\widetilde{C}(t) \widetilde{D}(t)
$$

where $\widetilde{C}(t)=e^{i\left(2 \pi v_{0} t\right)}$ and $\widetilde{D}(t)=e^{i \varphi(t)}$

By using the "Convolution Property" given in Appendix A we obtain

$$
W_{\widetilde{r}}(t, f)=\int_{-\infty}^{\infty} W_{\widetilde{C}}\left(t, f^{\prime}\right) W_{\widetilde{D}}\left(t, f-f^{\prime}\right) d f^{\prime}
$$

where $W_{\widetilde{C}}(t, f)$ and $W_{\widetilde{D}}(t, f)$ are the WDs of $\widetilde{C}(t)$ and $\widetilde{D}(t)$, respectively.

Note that the closed form expression for $W_{\widetilde{C}}\left(t, f^{\prime}\right)=$ $W_{\widetilde{s}}\left(t, f^{\prime}\right)$, where $W_{\widetilde{s}}\left(t, f^{\prime}\right)$ is given in (14) for $f^{\prime}=f$.

By substituting (14) into (16) we obtain

$$
\begin{aligned}
W_{\widetilde{r}}(t, f) & =\int_{-\infty}^{\infty} \delta\left(f^{\prime}-v_{0}\right) W_{D}\left(t, f-f^{\prime}\right) d f^{\prime} \\
& =W_{D}\left(t, f-v_{0}\right) \\
& =\int_{-\infty}^{\infty} e^{i \varphi(t+\tau / 2)} e^{-i \varphi(t-\tau / 2)} e^{-i 2 \pi\left(f-v_{0}\right) \tau} d \tau
\end{aligned}
$$

Let us define for simplicity the variable $\Delta \varphi(\tau)$ as

$$
\Delta \varphi(\tau)=\varphi\left(t-\frac{\tau}{2}\right)-\varphi\left(t+\frac{\tau}{2}\right)
$$

Thus, (17) can be written as

$$
\begin{aligned}
W_{\widetilde{r}}(t, f) & =W_{D}\left(t, f-v_{0}\right) \\
& =\int_{-\infty}^{\infty} e^{-i \Delta \varphi(\tau)} e^{-i 2 \pi\left(f-\nu_{0}\right) \tau} d \tau
\end{aligned}
$$

Now we turn to the "cross-term" component of $W_{\widetilde{r}-\widetilde{s}}(t, f)$ which is the third component on the right side of (13):

$$
\begin{aligned}
& W_{\tilde{r}, \widetilde{s}}(t, f) \\
& \quad=\int_{-\infty}^{\infty} e^{i\left[2 \pi v_{0}(t+\tau / 2)+\varphi(t+\tau / 2)\right]} e^{-i\left[2 \pi v_{0}(t-\tau / 2)\right]} e^{-i 2 \pi f \tau} d \tau \\
& \quad=\int_{-\infty}^{\infty} e^{i\left[\left(2 \pi v_{0} \tau\right)+\varphi(t+\tau / 2)\right]} e^{-i 2 \pi f \tau} d \tau
\end{aligned}
$$

Using (13), (17), and (20), the WD of (12) is given by

$$
\begin{aligned}
W_{\tilde{r}-\tilde{s}}(t, f)=\delta & \left(f-\nu_{0}\right) \\
& +\int_{-\infty}^{\infty} e^{i \varphi(t+\tau / 2)} e^{-i \varphi(t-\tau / 2)} e^{-i 2 \pi\left(f-\nu_{0}\right) \tau} d \tau \\
& -2 \operatorname{Re}\left\{\int_{-\infty}^{\infty} e^{i\left[\left(2 \pi v_{0} \tau\right)+\varphi(t+\tau / 2)\right]} e^{-i 2 \pi f \tau} d \tau\right\}
\end{aligned}
$$

This completes our proof of Theorem 1 .

\section{Constant Phase Offset}

As we all know, modern signal processing uses the discrete form of physical signals. Therefore, the practical computation will use the discrete Wigner-Ville distribution form as is introduced later in this subsection. Due to the simplicity of the mathematical development of using the continuous form of the WD upon its discrete one, we will use henceforth in our development process only the continuous form. In order to do so, we need to verify that there is a complete match between the analytic continuous form and the discrete one. Hence, in this subsection we will derive the analytic discrete form for the Wigner-Ville distribution and its TimeMarginal expression for a constant phase offset. In the end of the discrete form development, we will compare the discrete expression against the continuous expression introduced in the last subsection in order to verify a complete match and hence use only the continuous form.

4.1. Continuous Form. In this subsection, we will apply the analytic continuous form of the Wigner-Ville distribution on $\widetilde{r}-\widetilde{s}$ and then we will derive its Time-Marginal expression for a constant phase offset case.

Theorem 2. For the following assumptions:

(1) without loss of generality, $A=A_{0}=1$,

(2) $x_{0}$ is the constant phase offset from a reference clock signal,

(3) $v_{0}$ is the nominal frequency,

(4) $\widetilde{s}(t)$ denotes the continuous complex form of an ideal clock signal,

(5) $\tilde{r}_{1}(t)$ denotes the continuous complex form of the real clock signal for the case of constant phase offset. 
The Time-Marginal expression of the Wigner-Ville distribution for $\widetilde{r}_{1}(t)-\widetilde{s}(t)$ is defined as

$$
\left\|\widetilde{r}_{1}(t)-\widetilde{s}(t)\right\|^{2}=2\left\{1-\cos \left[2 \pi v_{0} x_{0}\right]\right\}
$$

Proof of Theorem 2. For simplicity in the following we denote $\widetilde{r}(t)$ as $\widetilde{r}_{1}(t)$.

The complex form of a clock signal with constant phase offset is given by

$$
\tilde{r}_{1}(t)=e^{i\left[2 \pi v_{0} t+\varphi_{1}(t)\right]}
$$

where the phase variation considering the constant phase is defined by

$$
\varphi_{1}(t)=2 \pi v_{0} x_{0}
$$

The terms presented in (18) are given by

$$
\varphi_{1}\left(t+\frac{\tau}{2}\right)=\varphi_{1}\left(t-\frac{\tau}{2}\right)=2 \pi v_{0} x_{0}
$$

By substituting (25) into (17) we obtain

$$
W_{\widetilde{r}_{1}}(t, f)=\int_{-\infty}^{\infty} e^{-i 2 \pi\left(f-v_{0}\right) \tau} d \tau=\delta\left(f-v_{0}\right)
$$

By substituting (25) into (20) we obtain

$$
\begin{aligned}
W_{\widetilde{r}_{1}, \widetilde{s}}(t, f) & =\int_{-\infty}^{\infty} e^{i\left[\left(2 \pi v_{0} \tau\right)+\varphi_{1}(t+\tau / 2)\right]} e^{-i 2 \pi f \tau} d \tau \\
& =\int_{-\infty}^{\infty} e^{i\left[\left(2 \pi v_{0} \tau\right)+2 \pi v_{0} x_{0}\right]} e^{-i 2 \pi f \tau} d \tau \\
& =e^{i 2 \pi v_{0} x_{0}} \delta\left(f-v_{0}\right)
\end{aligned}
$$

Then, the real part of $W_{\widetilde{r}_{1}, \widetilde{s}}(t, f)$ is

$$
\operatorname{Re}\left\{W_{\widetilde{r}_{1}, \widetilde{s}}(t, f)\right\}=\cos \left[2 \pi v_{0} x_{0}\right] \delta\left(f-v_{0}\right)
$$

By substituting (14), (26), and (28) into (13) we obtain

$$
\begin{aligned}
W_{\widetilde{r}_{1}-\widetilde{s}}(t, f)= & W_{\widetilde{r}_{1}}(t, f)+W_{\widetilde{s}}(t, f) \\
& -2 \operatorname{Re}\left\{W_{\widetilde{r}_{1}, \widetilde{s}}(t, f)\right\} \\
= & 2 \delta\left(f-v_{0}\right)\left\{1-\cos \left[2 \pi v_{0} x_{0}\right]\right\}
\end{aligned}
$$

Let us derive a closed form expression for the Time Marginal of (29)

$$
\begin{aligned}
& \left\|\widetilde{r}_{1}(t)-\widetilde{s}(t)\right\|^{2}=\int_{-\infty}^{\infty} W_{\widetilde{r}_{1}-\widetilde{s}}(t, f) d f \\
& \quad=2\left\{1-\cos \left[2 \pi v_{0} x_{0}\right]\right\} \int_{-\infty}^{\infty} \delta\left(f-v_{0}\right) d f \\
& \quad=2\left\{1-\cos \left[2 \pi v_{0} x_{0}\right]\right\}
\end{aligned}
$$

This completes our proof of Theorem 2.

\subsection{Discrete Form}

Theorem 3. For the following assumptions:

(1) without loss of generality, $A=A_{0}=1$,

(2) $x_{0}$ is the constant phase offset from a reference clock signal,

(3) $v_{0}$ is the nominal frequency,

(4) $\widetilde{s}[n]$ denotes the discrete complex form of an ideal clock signal,

(5) $\widetilde{r}_{1}[n]$ denotes the discrete complex form of the real clock signal for the case of constant phase offset.

The discrete Time-Marginal expression of the WignerVille distribution for $\widetilde{r}_{1}[n]-\widetilde{s}[n]$ is defined as

$$
\left\|\widetilde{r}_{1}[n]-\widetilde{s}[n]\right\|^{2}=2\left\{1-\cos \left[2 \pi v_{0} x_{0}\right]\right\}
$$

Proof of Theorem 3. For discrete time and frequency variables the Wigner distribution is defined as [58]

$$
w_{\tilde{r}}(n, m)=t_{s} \sum_{k=-k_{n}}^{k_{n}} \tilde{r}\left[n+\frac{k}{2}\right] \widetilde{r}^{*}\left[n-\frac{k}{2}\right] e^{-i 2 \pi m k /(2 N)}
$$

where $k_{n}=\min [2 n, 2 N-1-2 n], N$ denotes the number of samples, and $n, m$ denotes the index numbers for the time and frequency vectors, respectively.

Let us derive a closed form expression for the discrete Wigner-Ville distribution (DWD) of $\widetilde{r}-\widetilde{s}$ :

$$
w_{\widetilde{r}-\widetilde{s}}(n, m)=D W D\left[e^{i\left(2 \pi v_{0} n+\varphi[n]\right)}-e^{i\left(2 \pi v_{0} n\right)}\right]
$$

By using the "Summation Property" given in Appendix A (A.2) we obtain

$$
\begin{aligned}
w_{\widetilde{r}-\widetilde{s}}(n, m)= & w_{\widetilde{r}}(n, m)+w_{\widetilde{s}}(n, m) \\
& -2 \operatorname{Re}\left\{w_{\widetilde{r}, \widetilde{s}}(n, m)\right\}
\end{aligned}
$$

Let us get first a closed form expression for the second component on the right side of (34)

$$
\begin{aligned}
& w_{\widetilde{s}}(n, m)=t_{s} \sum_{k=-k_{n}}^{k_{n}} \widetilde{s}\left[n+\frac{k}{2}\right] \widetilde{s}^{*}\left[n-\frac{k}{2}\right] e^{-i 2 \pi m k /(2 N)} \\
& =t_{s} \sum_{k=-k_{n}}^{k_{n}} e^{i\left[2 \pi v_{0}(n+k / 2)\right]} e^{-i\left[2 \pi v_{0}(n-k / 2)\right]} e^{-i 2 \pi m k /(2 N)} \\
& =t_{s} \sum_{k=-k_{n}}^{k_{n}} e^{-i 2 \pi\left(m-2 N v_{0}\right) k /(2 N)}=t_{s} \delta\left(m-2 N v_{0}\right)
\end{aligned}
$$


Let us derive the first component on the right side of (34)

$$
\begin{aligned}
& w_{\widetilde{r}_{1}}(n, m)=t_{s} \sum_{k=-k_{n}}^{k_{n}} \widetilde{r}_{2}\left[n+\frac{k}{2}\right] \widetilde{r}_{2}\left[n-\frac{k}{2}\right] e^{-i 2 \pi m k /(2 N)} \\
& =t_{s} \sum_{k=-k_{n}}^{k_{n}} e^{i\left[2 \pi v_{0}(n+k / 2)+\varphi_{1}[n+k / 2]\right]} e^{-i\left[2 \pi v_{0}(n-k / 2)+\varphi_{1}[n-k / 2]\right]} e^{-i 2 \pi m k /(2 N)} \\
& =t_{s} \sum_{k=-k_{n}}^{k_{n}} e^{-i 2 \pi\left(m-2 N v_{0}\right) k /(2 N)}=t_{s} \delta\left(m-2 N v_{0}\right)
\end{aligned}
$$

Now we turn to the "cross-term" component of $w_{\widetilde{r}-\widetilde{s}}(n, m)$ which is the third component on the right side of (34).

The complex form of a discrete clock signal with constant phase offset is given by

$$
\widetilde{r}_{1}[n]=e^{i\left(2 \pi v_{0} n+\varphi[n]\right)}
$$

where the constant phase is defined by

$$
\varphi_{1}[n]=2 \pi v_{0} x_{0}
$$

The term presented in (18) is given by

$$
\varphi_{1}\left[n+\frac{k}{2}\right]=2 \pi v_{0} x_{0}
$$

Then, the cross-term derivation is given by

$$
\begin{aligned}
& w_{\tilde{r}, \tilde{s}}(n, m) \\
& =t_{s} \sum_{k=-k_{n}}^{k_{n}} e^{-i 2 \pi m k /(2 N)} e^{i\left[2 \pi v_{0}(n+k / 2)+\varphi[n+k / 2]\right]} e^{-i\left[2 \pi v_{0}(n-k / 2)\right]} \\
& =t_{s} \sum_{k=-k_{n}}^{k_{n}} e^{i\left[\left(2 \pi v_{0} k\right)+\varphi[n+k / 2]\right]} e^{-i 2 \pi m k /(2 N)}
\end{aligned}
$$

By substituting (39) into (40) we obtain

$$
\begin{aligned}
w_{\widetilde{r}_{1}, \widetilde{s}}(n, m) & =t_{s} \sum_{k=-k_{n}}^{k_{n}} e^{i\left[\left(2 \pi v_{0} k\right)+\varphi[n+k / 2]\right]} e^{-i 2 \pi m k /(2 N)} \\
& =t_{s} \sum_{k=-k_{n}}^{k_{n}} e^{i\left[\left(2 \pi v_{0} k\right)+2 \pi v_{0} x_{0}\right]} e^{-i 2 \pi m k /(2 N)} \\
& =e^{i 2 \pi v_{0} x_{0}} t_{s} \delta\left(m-2 N v_{0}\right)
\end{aligned}
$$

Then, the real part of $w_{\widetilde{r}_{1}}, \widetilde{s}(n, m)$ is

$$
\operatorname{Re}\left\{w_{\widetilde{r}_{1}, \widetilde{s}}(n, m)\right\}=\cos \left[2 \pi v_{0} x_{0}\right] t_{s} \delta\left(m-2 N v_{0}\right)
$$

By substituting (35), (36), and (42) into (34) we obtain

$$
w_{\widetilde{r}_{1}-\widetilde{s}}(n, m)=2 t_{s} \delta\left(m-2 N v_{0}\right)\left\{1-\cos \left[2 \pi v_{0} x_{0}\right]\right\}
$$

Finally, let us drive the closed form expression for the Time Marginal of (43)

$$
\begin{aligned}
\left\|\widetilde{r}_{1}[n]-\widetilde{s}[n]\right\|^{2}=\frac{f_{s}}{2 N} \sum_{m=0}^{2 N-1} w_{\widetilde{r}_{1}-\widetilde{s}}(n, m) \\
=\frac{f_{s}}{2 N} 2 t_{s}\left\{1-\cos \left[2 \pi v_{0} x_{0}\right]\right\} \sum_{m=0}^{2 N-1} \delta\left(m-2 N v_{0}\right) \\
=\frac{f_{s}}{2 N} 2 t_{s}\left\{1-\cos \left[2 \pi v_{0} x_{0}\right]\right\} 2 N \\
=2\left\{1-\cos \left[2 \pi v_{0} x_{0}\right]\right\}
\end{aligned}
$$

This completes our proof of Theorem 3.

As can be seen clearly, the discrete expression is the same as the continuous expression (22) introduced in the last subsection. This means that henceforth we can use only the continuous form and it will describe correctly the practical discrete signal processing.

\section{Constant Frequency Offset}

In this section, we will derive the analytic continuous form for the Wigner-Ville distribution and its Time-Marginal expression for the frequency offset case in addition to the phase offset case.

Theorem 4. For the following assumptions:

(1) without loss of generality, $A=A_{0}=1$,

(2) $x_{0}$ is the constant phase offset from a reference clock signal,

(3) $y_{0}$ is the constant fractional frequency offset from a reference clock signal,

(4) $v_{0}$ is the nominal frequency,

(5) $\widetilde{s}(t)$ denotes the continuous complex form of an ideal clock signal,

(6) $\tilde{r}_{2}(t)$ denotes the continuous complex form of the real clock signal for the case of constant phase and frequency offsets.

The Time-Marginal expression of the Wigner-Ville distribution for $\widetilde{r}_{2}(t)-\widetilde{s}(t)$ is defined as

$$
\left\|\widetilde{r}_{2}(t)-\widetilde{s}(t)\right\|^{2}=2\left\{1-\cos \left[2 \pi v_{0}\left(x_{0}+y_{0} t\right)\right]\right\}
$$

Proof of Theorem 4. For simplicity in the following we denote $\widetilde{r}(t)$ as $\widetilde{r}_{2}(t)$.

The complex form of a clock signal with constant phase and frequency offsets is given by

$$
\widetilde{r}_{2}(t)=e^{i\left[2 \pi v_{0} t+\varphi_{2}(t)\right]}
$$

where the phase variation considering the constant phase and frequency offsets is defined by

$$
\varphi_{2}(t)=2 \pi v_{0}\left(x_{0}+y_{0} t\right)
$$


where we may define

$$
\varphi_{2}\left(t \pm \frac{\tau}{2}\right)=2 \pi v_{0}\left[x_{0}+y_{0}\left(t \pm \frac{\tau}{2}\right)\right]
$$

By substituting (48) into (18) we obtain

$$
\Delta \varphi_{2}(\tau)=\varphi_{2}\left(t-\frac{\tau}{2}\right)-\varphi_{2}\left(t+\frac{\tau}{2}\right)=2 \pi v_{0}\left(-y_{0} \tau\right)
$$

In order to carry out the first term in the right side of (12), the expression in (49) is substituted into (19):

$$
\begin{aligned}
W_{\widetilde{r}_{2}}(t, f) & =\int_{-\infty}^{\infty} e^{-i \Delta \varphi_{2}(\tau)-i 2 \pi\left(f-\nu_{0}\right) \tau} d \tau \\
& =\int_{-\infty}^{\infty} e^{-i\left[2 \pi v_{0}\left(-y_{0} \tau\right)\right]-i 2 \pi\left(f-v_{0}\right) \tau} d \tau \\
& =\delta\left[f-v_{0}\left(y_{0}+1\right)\right]
\end{aligned}
$$

Next, in order to carry out the third term in the right side of (12), the expression in (48) is substituted into (20):

$$
\begin{aligned}
W_{\widetilde{r}_{2}, \widetilde{s}}(t, f) & =\int_{-\infty}^{\infty} e^{i\left[2 \pi v_{0} \tau+\varphi_{2}(t+\tau / 2)\right]} e^{-i 2 \pi f \tau} d \tau \\
& =\int_{-\infty}^{\infty} e^{i\left\{2 \pi v_{0} \tau+2 \pi v_{0}\left[x_{0}+y_{0}(t+\tau / 2)\right]\right\}} e^{-i 2 \pi f \tau} d \tau \\
& =e^{i 2 \pi v_{0}\left(x_{0}+y_{0} t\right)} \delta\left[f-v_{0}\left(\frac{y_{0}}{2}+1\right)\right]
\end{aligned}
$$

Then, the real part of (51) is

$$
\begin{aligned}
\operatorname{Re} & \left\{W_{\widetilde{r}_{2}, \widetilde{s}}(t, f)\right\} \\
& =\cos \left[2 \pi v_{0}\left(x_{0}+y_{0} t\right)\right] \delta\left[f-v_{0}\left(\frac{y_{0}}{2}+1\right)\right]
\end{aligned}
$$

By substituting (14), (50), and (52) into (13) we obtain

$$
\begin{aligned}
W_{\widetilde{r}_{2}-\widetilde{s}} & (t, f) \\
= & \delta\left[f-v_{0}\left(y_{0}+1\right)\right]+\delta\left(f-v_{0}\right) \\
& \quad-2 \cos \left[2 \pi v_{0}\left(x_{0}+y_{0} t\right)\right] \delta\left[f-v_{0}\left(\frac{y_{0}}{2}+1\right)\right]
\end{aligned}
$$

Now we turn to derive the Time-Marginal expression of (53)

$$
\begin{aligned}
& \left\|\widetilde{r}_{2}(t)-\widetilde{s}(t)\right\|^{2}=\int_{-\infty}^{\infty} W_{\widetilde{r}_{2}-\widetilde{s}}(t, f) d f \\
& =\int_{-\infty}^{\infty} \delta\left[f-v_{0}\left(y_{0}+1\right)\right] d f \\
& \quad+\int_{-\infty}^{\infty} \delta\left(f-v_{0}\right) d f-\int_{-\infty}^{\infty} 2 \\
& \quad \cdot \cos \left[2 \pi v_{0}\left(x_{0}+y_{0} t\right)\right] \delta\left[f-v_{0}\left(\frac{y_{0}}{2}+1\right)\right] d f \\
& \quad=2\left\{1-\cos \left[2 \pi v_{0}\left(x_{0}+y_{0} t\right)\right]\right\}
\end{aligned}
$$

This completes our proof of Theorem 4 .

As we can see the result that we get is a periodic signal with a linear period of $1 /\left[v_{0}\left(x_{0}+y_{0} t\right)\right]$. In Section 7 , we will examine the analytic expression versus the numerical simulation results in order to verify our expression.

\section{Frequency Drift}

Now we derive the analytic continuous form for the WignerVille distribution and its Time-Marginal expression for the case of frequency drift along with constant phase and frequency offsets.

Theorem 5. For the following assumptions:

(1) without loss of generality, $A=A_{0}=1$,

(2) $x_{0}$ is the constant phase offset from a reference clock signal,

(3) $y_{0}$ is the constant fractional frequency offset from a reference clock signal,

(4) $v_{0}$ is the nominal frequency,

(5) $a$ is the linear frequency drift coefficient $(a \neq 0)$,

(6) $\widetilde{s}(t)$ denotes the continuous complex form of an ideal clock signal,

(7) $\tilde{r}_{3}(t)$ denotes the continuous complex form of the real clock signal for the case of frequency drift.

The Time-Marginal expression of the Wigner-Ville distribution for $\left[\widetilde{r}_{3}(t)-\widetilde{s}(t)\right]$ is defined as

For $a<0$ :

$$
\begin{aligned}
& \left\|\widetilde{r}_{3}(t)-\widetilde{s}(t)\right\|^{2}=2 \\
& -2 \sqrt{\frac{4}{v_{0}|a|}} \int_{-\infty}^{\infty} \cos \left\{\frac{4 \pi}{v_{0}|a|}\left[f-v_{0}\left(\frac{a}{2} t+\frac{y_{0}}{2}+1\right)\right]^{2}\right. \\
& \left.-\frac{\pi}{4}+2 \pi v_{0}\left(x_{0}+y_{0} t+a \frac{t^{2}}{2}\right)\right\} d f
\end{aligned}
$$

For $a>0$ :

$$
\begin{aligned}
& \left\|\widetilde{r}_{3}(t)-\widetilde{s}(t)\right\|^{2}=2 \\
& -2 \sqrt{\frac{4}{v_{0}|a|}} \int_{-\infty}^{\infty} \cos \left\{-\frac{4 \pi}{v_{0}|a|}\left[f-v_{0}\left(\frac{a}{2} t+\frac{y_{0}}{2}+1\right)\right]^{2}\right. \\
& \left.\quad-\frac{\pi}{4}+2 \pi v_{0}\left(x_{0}+y_{0} t+a \frac{t^{2}}{2}\right)\right\} d f
\end{aligned}
$$

Proof of Theorem 5. For simplicity in the following we denote $\widetilde{r}(t)$ as $\widetilde{r}_{3}(t)$.

The complex form of a clock signal with frequency drift along with constant phase and frequency offsets is given by

$$
\widetilde{r}_{3}(t)=e^{i\left[2 \pi v_{0} t+\varphi_{3}(t)\right]}
$$

where the phase $\varphi_{3}(t)$ is defined by

$$
\begin{aligned}
\varphi_{3}(t) & =\omega_{0}\left[x_{0}+y_{0} t+a \frac{t^{2}}{2}\right] \\
& =2 \pi v_{0}\left[x_{0}+y_{0} t+a \frac{t^{2}}{2}\right]
\end{aligned}
$$


Based on (57), $\varphi_{3}(t \pm \tau / 2)$ can be written as

$$
\varphi_{3}\left(t \pm \frac{\tau}{2}\right)=2 \pi v_{0}\left[x_{0}+y_{0}\left(t \pm \frac{\tau}{2}\right)+\frac{a}{2}\left(t \pm \frac{\tau}{2}\right)^{2}\right]
$$

Now, by substituting (58) into (18) we get

$$
\begin{aligned}
\Delta \varphi_{3}(\tau) & =\varphi_{3}\left(t-\frac{\tau}{2}\right)-\varphi_{3}\left(t+\frac{\tau}{2}\right) \\
& =2 \pi v_{0}\left(-y_{0} \tau-a t \tau\right)
\end{aligned}
$$

Next, by substituting (59) into (17) we obtain

$$
\begin{aligned}
W_{\widetilde{r}_{3}}(t, f) & =\int_{-\infty}^{\infty} e^{-i \Delta_{\varphi_{3}}(\tau)-i 2 \pi\left(f-v_{0}\right) \tau} d \tau \\
& =\int_{-\infty}^{\infty} e^{-i\left[2 \pi v_{0}\left(-y_{0} \tau-a t \tau\right)\right]-i 2 \pi\left(f-v_{0}\right) \tau} d \tau \\
& =\delta\left[f-v_{0}\left(y_{0}+a t+1\right)\right]
\end{aligned}
$$

Substituting (58) into (20) we obtain

$$
\begin{aligned}
& W_{\widetilde{r}_{3} \widetilde{s}}(t, f)=-\int_{-\infty}^{\infty} e^{i\left[\omega_{0} \tau+\varphi_{3}(t+\tau / 2)\right]} e^{-i 2 \pi f \tau} d \tau \\
& =-\int_{-\infty}^{\infty} e^{i\left\{\omega_{0} \tau+2 \pi v_{0}\left[x_{0}+y_{0}(t+\tau / 2)+a\left((t+\tau / 2)^{2} / 2\right)\right]\right\}} e^{-i 2 \pi f \tau} d \tau \\
& =-e^{i 2 \pi v_{0}\left(x_{0}+y_{0} t+a\left(t^{2} / 2\right)\right)} F\left\{e^{i\left\{\omega_{0} \tau+2 \pi v_{0}\left(y_{0} \tau / 2+a(t \tau / 2)\right)\right\}} e^{i 2 \pi v_{0} a\left(\tau^{2} / 8\right)}\right\}
\end{aligned}
$$

where $F\{A\}$ denotes the Fourier transform of $A$.

In the following we derive the outcome of $F\left\{e^{i\left\{\omega_{0} \tau+2 \pi v_{0}\left(y_{0} \tau / 2+a(t \tau / 2)\right)\right\}} e^{i 2 \pi v_{0} a\left(\tau^{2} / 8\right)}\right\}$. For that purpose we define

$$
\begin{aligned}
& g(\tau)=\exp \left\{i\left[\omega_{0} \tau+2 \pi v_{0}\left(\frac{y_{0} \tau}{2}+a \frac{t \tau}{2}\right)\right]\right\} \\
& h(\tau)=\exp \left\{i 2 \pi v_{0} a \frac{\tau^{2}}{8}\right\}
\end{aligned}
$$

The Fourier transform of a product is defined by [59]

$$
F\{g(\tau) h(\tau)\}=G(f) * H(f)
$$

where $*$ denotes convolution.

Based on (62), $G(f)$ can be written as

$$
\begin{aligned}
G(f) & =\int_{-\infty}^{\infty} e^{i\left\{\omega_{0} \tau+2 \pi v_{0}\left(y_{0} \tau / 2+a(t \tau / 2)\right)\right\}} e^{-i 2 \pi f \tau} d \tau \\
& =\int_{-\infty}^{\infty} e^{-i 2 \pi\left\{f+v_{0}\left(-y_{0} / 2-a(t / 2)-1\right)\right\} \tau} d \tau \\
& =\delta\left[f-v_{0}\left(\frac{y_{0}}{2}+\frac{a t}{2}+1\right)\right]
\end{aligned}
$$

Based on (63), $H(f)$ can be defined as

$$
H(f)=\int_{-\infty}^{\infty} e^{i 2 \pi v_{0} a\left(\left(\tau^{2}\right) / 8\right)} e^{-i 2 \pi f \tau} d \tau
$$

The Fourier transform of a complex Gaussian is given by [59]

For $k>0$ :

$$
F\left\{e^{-i k \tau^{2}}\right\}=\sqrt{\frac{\pi}{k}} \exp \left\{i\left(\frac{\pi^{2} f^{2}}{k}-\frac{\pi}{4}\right)\right\}
$$

For $k<0$ :

$$
\begin{aligned}
F\left\{e^{-i k \tau^{2}}\right\} & =\sqrt{\frac{\pi}{|k|}} \exp \left\{-i\left(\frac{\pi^{2} f^{2}}{|k|}-\frac{\pi}{4}\right)\right\} \\
\text { For } k & =0 \\
F\left\{e^{-i k \tau^{2}}\right\} & =\delta(f)
\end{aligned}
$$

Based on (66) and (67) the expression for $k$ is given by $k=$ $-\pi v_{0} a / 4$.

Thus, based on (66) and (67) and that $k=-\pi v_{0} a / 4$ we have

$$
H(f)=\int_{-\infty}^{\infty} e^{i 2 \pi v_{0} a\left(\tau^{2} / 8\right)} e^{-i 2 \pi f \tau} d \tau=
$$

For $a<0$ :

$$
H(f)=\sqrt{\frac{4}{-v_{0} a}} \exp \left\{i\left(\frac{4 \pi f^{2}}{-\nu_{0} a}-\frac{\pi}{4}\right)\right\}
$$

For $a>0$ :

$$
H(f)=\sqrt{\frac{\pi}{\left|-\pi v_{0} a / 4\right|}} \exp \left\{-i\left(\frac{\pi^{2} f^{2}}{\left|-\pi v_{0} a / 4\right|}-\frac{\pi}{4}\right)\right\}
$$

By substituting (65) and (68) into (64) we obtain

For $a<0$ :

$$
\begin{aligned}
F\{g(\tau) h(\tau)\}= & \delta\left[f-v_{0}\left(\frac{y_{0}}{2}+\frac{a t}{2}+1\right)\right] \\
& * \sqrt{\frac{4}{v_{0}|a|}} \exp \left\{i\left(\frac{4 \pi f^{2}}{v_{0}|a|}-\frac{\pi}{4}\right)\right\}
\end{aligned}
$$

For $a>0$ :

$$
\begin{aligned}
F\{g(\tau) h(\tau)\}= & \delta\left[f-v_{0}\left(\frac{y_{0}}{2}+\frac{a t}{2}+1\right)\right] \\
& * \sqrt{\frac{4}{v_{0}|a|}} \exp \left\{-i\left(\frac{4 \pi f^{2}}{v_{0}|a|}-\frac{\pi}{4}\right)\right\}
\end{aligned}
$$

We use the following property [59]:

$$
G(f) * H(f)=\int_{-\infty}^{\infty} G(\rho) H(f-\rho) d \rho
$$


Next, by substituting (69) into (70) we obtain

For $a<0$ :

$$
G(f) * H(f)=\int_{-\infty}^{\infty} \delta\left[\rho-v_{0}\left(\frac{y_{0}}{2}+\frac{a t}{2}+1\right)\right] \sqrt{\frac{4}{v_{0}|a|}} \exp \left\{i\left[\frac{4 \pi}{v_{0}|a|}(f-\rho)^{2}-\frac{\pi}{4}\right]\right\} d \rho
$$

For $a>0$ :

$$
G(f) * H(f)=\int_{-\infty}^{\infty} \delta\left[\rho-v_{0}\left(\frac{y_{0}}{2}+\frac{a t}{2}+1\right)\right] \sqrt{\frac{4}{v_{0}|a|}} \exp \left\{-i\left[\frac{4 \pi}{v_{0}|a|}(f-\rho)^{2}-\frac{\pi}{4}\right]\right\} d \rho
$$

After carrying out the integration in (71) we have

For $a<0$ :

$$
\begin{aligned}
& G(f) * H(f)=\sqrt{\frac{4}{v_{0}|a|}} \\
& \quad \cdot \exp \left\{i\left\{\frac{4 \pi}{v_{0}|a|}\left[f-v_{0}\left(\frac{y_{0}}{2}+\frac{a t}{2}+1\right)\right]^{2}-\frac{\pi}{4}\right\}\right\}
\end{aligned}
$$

For $a>0$ :

$$
\begin{aligned}
& G(f) * H(f)=\sqrt{\frac{4}{v_{0}|a|}} \\
& \quad \cdot \exp \left\{-i\left\{\frac{4 \pi}{v_{0}|a|}\left[f-v_{0}\left(\frac{y_{0}}{2}+\frac{a t}{2}+1\right)\right]^{2}-\frac{\pi}{4}\right\}\right\}
\end{aligned}
$$

By substituting (72) into (61) we obtain

For $a<0$ :

$$
\begin{aligned}
& W_{\widetilde{r}_{3}, \widetilde{s}}(t, f)=-e^{i 2 \pi v_{0}\left(x_{0}+y_{0} t+a\left(t^{2} / 2\right)\right)} \sqrt{\frac{4}{v_{0}|a|}} \\
& \cdot \exp \left\{i\left\{\frac{4 \pi}{v_{0}|a|}\left[f-v_{0}\left(\frac{y_{0}}{2}+\frac{a t}{2}+1\right)\right]^{2}-\frac{\pi}{4}\right\}\right\}
\end{aligned}
$$

For $a>0$ :

$$
\begin{aligned}
& W_{\widetilde{r}_{3}, \widetilde{s}}(t, f)=-e^{i 2 \pi v_{0}\left(x_{0}+y_{0} t+a\left(t^{2} / 2\right)\right)} \sqrt{\frac{4}{v_{0}|a|}} \\
& \cdot \exp \left\{-i\left\{\frac{4 \pi}{v_{0}|a|}\left[f-v_{0}\left(\frac{y_{0}}{2}+\frac{a t}{2}+1\right)\right]^{2}-\frac{\pi}{4}\right\}\right\}
\end{aligned}
$$

Then, the real part of $W_{\widetilde{r}_{3}, \widetilde{s}}(t, f)$ is

For $a<0$ :

$$
\begin{aligned}
& \operatorname{Re}\left\{W_{\widetilde{r}_{3}, \widetilde{s}}(t, f)\right\}=-\sqrt{\frac{4}{v_{0}|a|}} \\
& \quad \cdot \cos \left\{\frac{4 \pi}{v_{0}|a|}\left[f-v_{0}\left(\frac{a}{2} t+\frac{y_{0}}{2}+1\right)\right]^{2}-\frac{\pi}{4}\right.
\end{aligned}
$$

$$
\left.+2 \pi v_{0}\left(x_{0}+y_{0} t+a \frac{t^{2}}{2}\right)\right\}
$$

For $a>0$ :

$$
\begin{aligned}
& \operatorname{Re}\left\{W_{\widetilde{r}_{3}, \widetilde{s}}(t, f)\right\}=-\sqrt{\frac{4}{v_{0}|a|}} \\
& \cdot \cos \left\{-\frac{4 \pi}{v_{0}|a|}\left[f-v_{0}\left(\frac{a}{2} t+\frac{y_{0}}{2}+1\right)\right]^{2}-\frac{\pi}{4}\right. \\
& \left.+2 \pi v_{0}\left(x_{0}+y_{0} t+a \frac{t^{2}}{2}\right)\right\}
\end{aligned}
$$

By substituting (14), (60), and (74) into (13) we obtain

For $a<0$ :

$$
\begin{aligned}
& W_{\widetilde{r}_{3}-\widetilde{s}}(t, f)=\delta\left[f-v_{0}\left(y_{0}+a t+1\right)\right]+\delta\left(f-v_{0}\right) \\
& -2 \sqrt{\frac{4}{v_{0}|a|}} \cos \left\{\frac{4 \pi}{v_{0}|a|}\left[f-v_{0}\left(\frac{a}{2} t+\frac{y_{0}}{2}+1\right)\right]^{2}\right. \\
& \left.-\frac{\pi}{4}+2 \pi v_{0}\left(x_{0}+y_{0} t+a \frac{t^{2}}{2}\right)\right\}
\end{aligned}
$$

For $a>0$ :

$$
\begin{aligned}
& W_{\widetilde{r}_{3}-\widetilde{s}}(t, f)=\delta\left[f-v_{0}\left(y_{0}+a t+1\right)\right]+\delta\left(f-v_{0}\right) \\
& -2 \sqrt{\frac{4}{v_{0}|a|}} \cos \left\{-\frac{4 \pi}{v_{0}|a|}\left[f-v_{0}\left(\frac{a}{2} t+\frac{y_{0}}{2}+1\right)\right]^{2}\right. \\
& \left.-\frac{\pi}{4}+2 \pi v_{0}\left(x_{0}+y_{0} t+a \frac{t^{2}}{2}\right)\right\}
\end{aligned}
$$

Based on (8) and (75) we obtain

For $a<0$ :

$\left\|\widetilde{r}_{3}(t)-\widetilde{s}(t)\right\|^{2}=2$ 


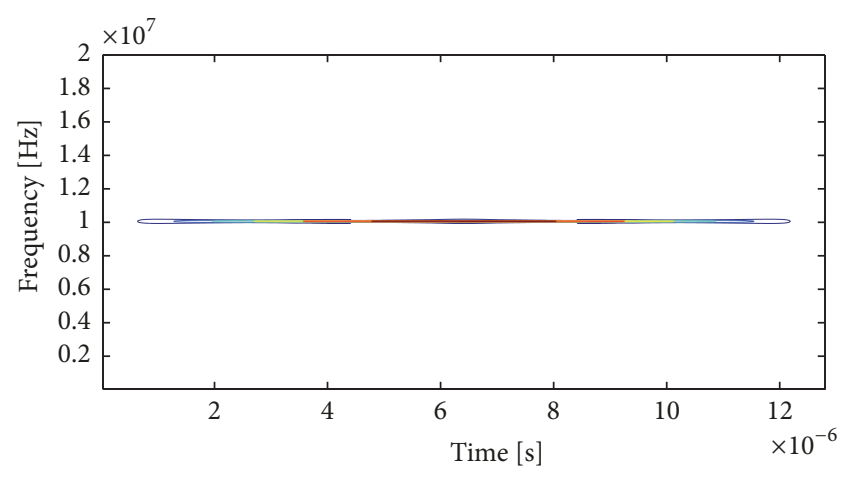

(a) WD of $10 \mathrm{MHz}$ Signal

FIgURE 2: The contour plot of a reference $10 \mathrm{MHz}$ Wigner-Ville distribution signal $\widetilde{s}(t)$. The sampling frequency is $40 \mathrm{MHz}$.

$$
\begin{aligned}
& -2 \sqrt{\frac{4}{v_{0}|a|}} \int_{-\infty}^{\infty} \cos \left\{\frac{4 \pi}{v_{0}|a|}\left[f-v_{0}\left(\frac{a}{2} t+\frac{y_{0}}{2}+1\right)\right]^{2}\right. \\
& \left.-\frac{\pi}{4}+2 \pi v_{0}\left(x_{0}+y_{0} t+a \frac{t^{2}}{2}\right)\right\} d f
\end{aligned}
$$

For $a>0$ :

$$
\begin{aligned}
& \left\|\widetilde{r}_{3}(t)-\widetilde{s}(t)\right\|^{2}=2 \\
& -2 \sqrt{\frac{4}{v_{0}|a|}} \int_{-\infty}^{\infty} \cos \left\{-\frac{4 \pi}{v_{0}|a|}\left[f-v_{0}\left(\frac{a}{2} t+\frac{y_{0}}{2}+1\right)\right]^{2}\right. \\
& \left.-\frac{\pi}{4}+2 \pi v_{0}\left(x_{0}+y_{0} t+a \frac{t^{2}}{2}\right)\right\} d f
\end{aligned}
$$

This completes our proof of Theorem 5.

\section{Numerical Simulations}

In this section, we will show the simulation results for the continuous and discrete Time-Marginal Wigner-Ville distribution of the differences $\left[\widetilde{r}_{1}(t)-\widetilde{s}(t)\right]$ and $\left[\widetilde{r}_{1}[n]-\widetilde{s}[n]\right]$ compared to our analytical proposed expressions (22) and (31), respectively. In order to demonstrate different nominal frequencies, we simulate the Time-Marginal Wigner-Ville distribution of the differences $\left[\widetilde{r}_{1}(t)-\widetilde{s}(t)\right]$ for three different nominal frequencies.

Figure 2 shows the contour plot of the Wigner-Ville distribution of a reference $10 \mathrm{MHz}$ signal $\widetilde{s}(t)$, where the sampling frequency is $40 \mathrm{MHz}$.

Figures 3-5 show for three different values of $v_{0}$ the comparison between the outcomes of the following expressions, (22) and (31), which were derived for the continuous and discrete cases, respectively.

According to Figures 3-5, there are high correlation between the outcomes of (22) and (31).

Figures 6 and 7 show the contour plot of (53), where the upper, lower, and the middle frequencies lines match the first, second, and the third terms of (53), respectively.

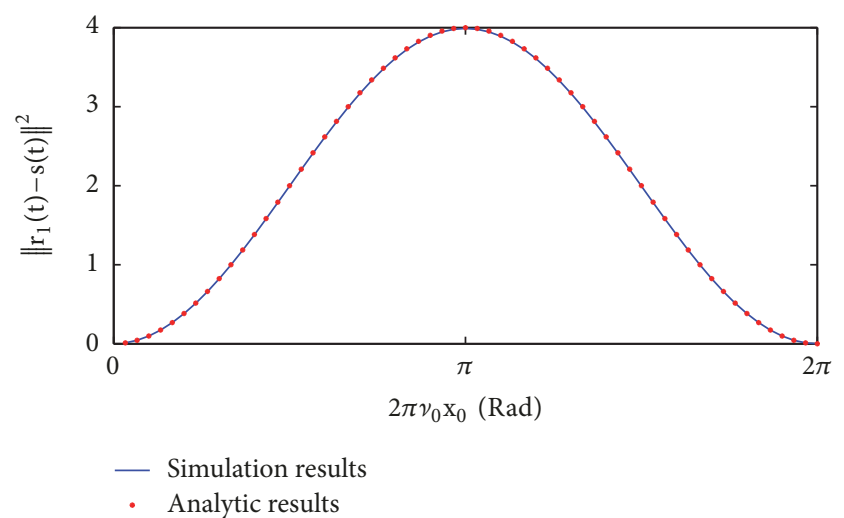

Figure 3: The Time-Marginal Wigner-Ville distribution $\left\|\widetilde{r}_{1}(t)-\widetilde{s}(t)\right\|$ versus the constant phase offset $\varphi_{1}(t)=2 \pi v_{0} x_{0}$. The nominal frequency $v_{0}$ is $10 \mathrm{MHz}$ and the sampling frequency is $40 \mathrm{MHz}$.

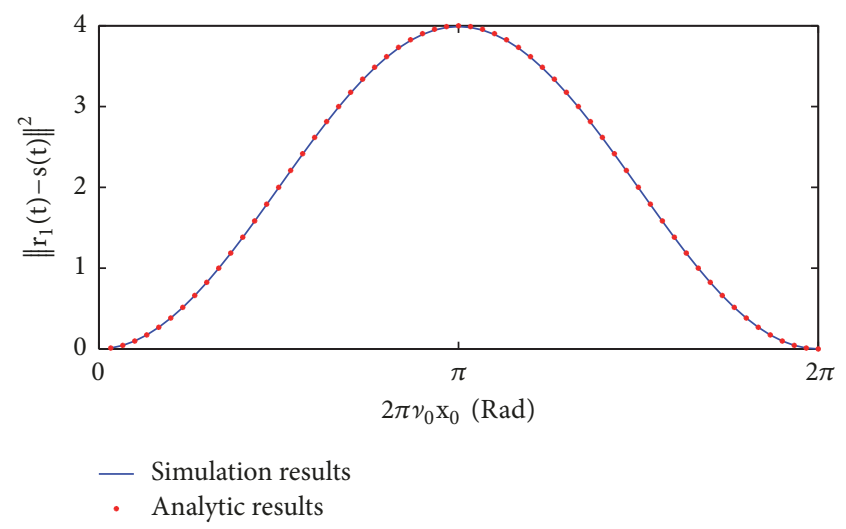

Figure 4: The Time-Marginal Wigner-Ville distribution $\left\|\widetilde{r}_{1}(t)-\widetilde{s}(t)\right\|$ versus the constant phase offset $\varphi_{1}(t)=2 \pi v_{0} x_{0}$. The nominal frequency $v_{0}$ is $5 \mathrm{MHz}$ and the sampling frequency is $20 \mathrm{MHz}$.

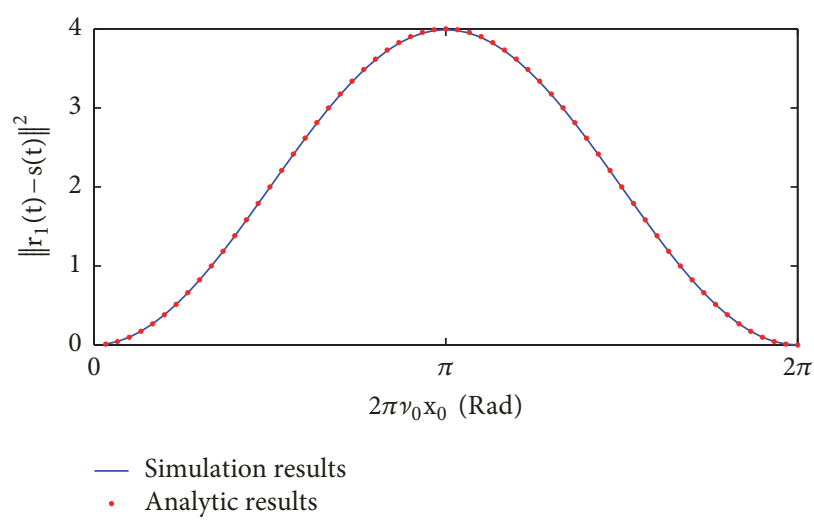

FIgURE 5: The Time-Marginal Wigner-Ville distribution $\left\|\widetilde{r}_{1}(t)-\widetilde{s}(t)\right\|$ versus the constant phase offset $\varphi_{1}(t)=2 \pi v_{0} x_{0}$. The nominal frequency $v_{0}$ is $20 \mathrm{MHz}$ and the sampling frequency is $80 \mathrm{MHz}$.

Figure 8 shows the contour plot of (75), for $a=-12 \mathrm{KHz}$, $y_{0}=0.15, v_{0}=10 \mathrm{MHz}$, and sampling frequency of $40 \mathrm{MHz}$. We can see clearly that the linear chirp line follows the first term of (75). The constant frequency line matches the second 


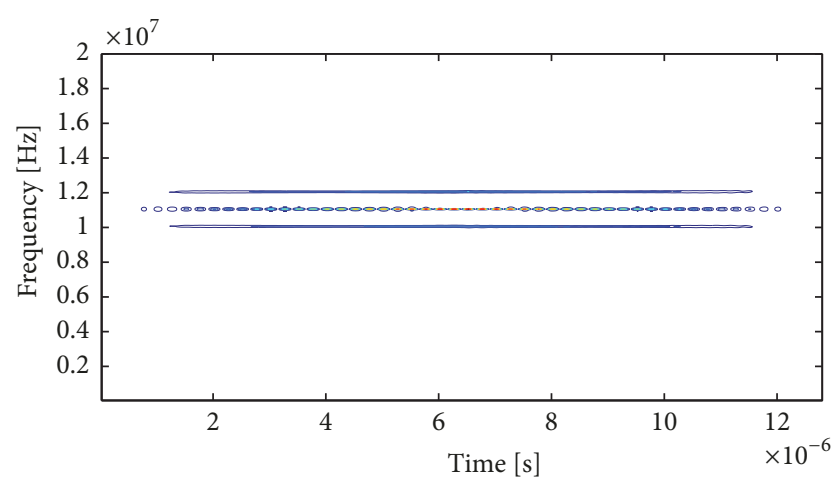

(a) WD of $10 \mathrm{MHz}$ Signal, $y(0)=0.2 \mathrm{MHz}$

Figure 6: The contour of the Wigner-Ville distribution of the difference $\widetilde{r}_{2}(t)-\widetilde{s}(t)$, where $y_{0}=0.2$. The nominal frequency $v_{0}$ is $10 \mathrm{MHz}$ and the sampling frequency is $40 \mathrm{MHz}$.

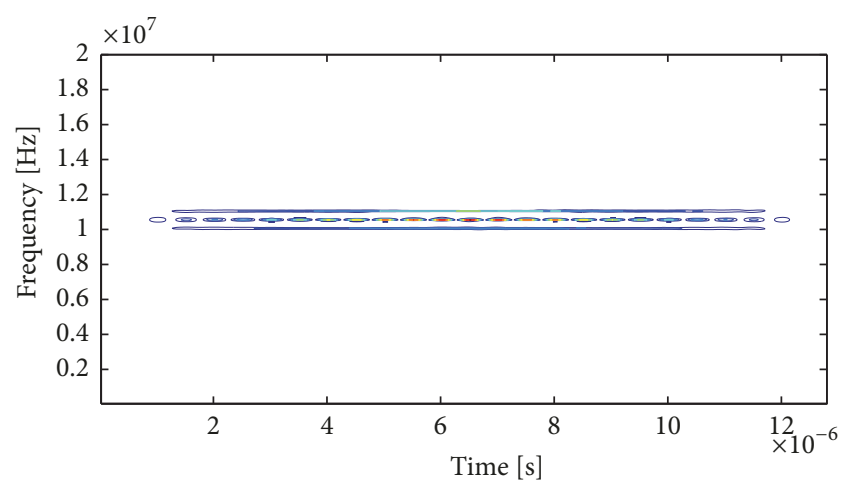

(a) WD of $10 \mathrm{MHz}$ Signal, $y(0)=0.1 \mathrm{MHz}$

FIgURE 7: The contour of the Wigner-Ville distribution of the difference $\widetilde{r}_{2}(t)-\widetilde{s}(t)$, where $y_{0}=0.1$. The nominal frequency $v_{0}$ is $10 \mathrm{MHz}$ and the sampling frequency is $40 \mathrm{MHz}$.

term of (75) and the cross-term frequencies (in the middle) correspond to the third term of (75).

\section{Summary and Conclusion}

In this paper, we have presented a new tool for frequency source characterization based on the Wigner-Ville distribution, whose objective is to represent the variation of clock noise when no stationarity assumption is made. This means that the new tool can be used for a nonstationary phase variation. We presented the analytic terms for the WignerVille distribution and its Time Marginal for constant phase and frequency offsets and frequency drift from a reference source. Simulation results indicate that our derivations hold.

\section{Appendix}

\section{A.}

In this appendix, we will present many meaningful mathematical properties of the Wigner distribution [33]. The mathematical properties can be summarized as follows:
(P1) $W_{s}(t, f)=W_{s}^{*}(t, f)$ (the WD is a strictly real-valued function).

(P2) If $s(t)=0 t<t_{a}$ or $t>t_{b}$ then $W_{s}(t, f)=0 t<t_{a}$ or $t>t_{b}$.

(P3) For real $s(t) W_{s}(t, f)=W_{s}(t,-f)$.

(P4) $\int_{-\infty}^{\infty} W_{s}(t, f) d f=\|s(t)\|^{2}$ (Time-Marginal property).

(P5) $\int_{-\infty}^{\infty} W_{s}(t, f) d t=\|S(f)\|^{2}(S(f)$ represents the FT of the function $s(t))$.

((P4) and (P5) are customarily referred as marginals).

(P6) If $g(t)=s\left(t-t_{0}\right)$, then $W_{g}(t, f)=W_{s}\left(t-t_{0}, f\right)$ (shift property).

(P7) If $g(t)=s(t) e^{i\left(f_{0} t\right)}$, then $W_{g}(t, f)=W_{s}\left(t, f-f_{0}\right)$ (modulation property).

(P8) If $g(t)=s(t) h(t)$, then $W_{g}(t, f)=W_{s}(t, f) * W_{h}(t, f)$ (convolution property).

(P9) $(1 / 2 \pi) \int_{-\infty}^{\infty} W_{s}(t / 2, f) e^{i f t} d f=s(t) s^{*}(0)$ (inversion property).

(P10) If $[60,61]$ : (summation property).

$$
g(t)=s(t)+h(t)
$$

Then, the Wigner-Ville distribution of $g(t)$ is given by

$$
W_{g}(t, f)=W_{s}(t, f)+W_{h}(t, f)+2 \operatorname{Re}\left\{W_{s, h}(t, f)\right\}
$$

where $W_{s}(t, f)$ and $W_{h}(t, f)$ are the Wigner-Ville distributions of $s(t)$ and $h(t)$, respectively, and refer to as "autoterms".

The cross-term is given by $[60,61]$

$$
\begin{aligned}
2 \operatorname{Re} & \left\{W_{s, h}(t, f)\right\} \\
& =2 \operatorname{Re}\left\{F\left[s\left(t+\frac{\tau}{2}\right) h^{*}\left(t-\frac{\tau}{2}\right)\right]\right\}
\end{aligned}
$$

The WD has an obvious interpretation as defining a local power spectrum at each point of time $t$ of the signal. Remarkably, property (P1) implies that the phase of the WD is implicit (phase remains hidden in the real-valued WD function).

Also, (P9) indicates that the WD is a reversible transform and the original signal can be recovered up to a constant factor $s^{*}(0)$ (Claasen and Mecklenbrauker, 1980) [55-57].

B.

In this Appendix, we present and explain in detail the discrete form of the Wigner distribution. Although the WD was initially defined for continuous variable functions, Claasen and Mecklenbrauker (1980) [55] proposed a first definition for discrete variable functions. However, some attempts to extend definitions of the WD to discrete signals have not yet been completely successful (O'Neill, Flandrin, and Williams, 1998) [62]. For the applications in this paper, the following discrete Wigner distribution, similar to the one proposed 


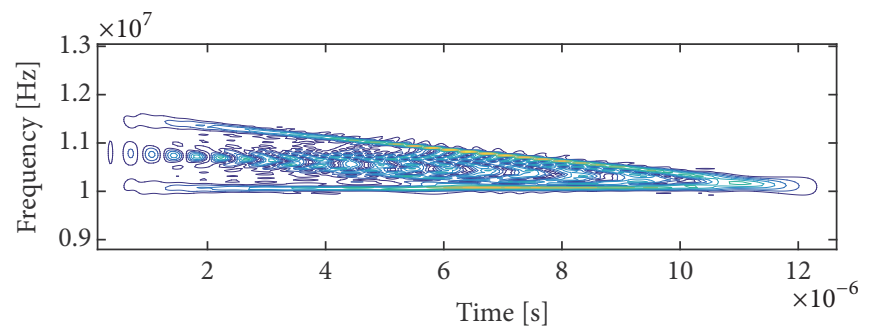

(9) Wigner distribution contour of a linear chirp minus a $10 \mathrm{MHz}$ sinusoid. $\mathrm{a}=-12 \mathrm{KHz}, \mathrm{y}(0)=0.15, \mathrm{x}(0)=0$

FIgURE 8: The contour of the Wigner-Ville distribution of the difference $\widetilde{r}_{3}(t)-\widetilde{s}(t)$, where $a=-12 K H z, y_{0}=0.15$, and $x_{0}=0$. The nominal frequency $v_{0}$ is $10 \mathrm{MHz}$ and the sampling frequency is $40 \mathrm{MHz}$.

by Eric Chassande-Mottin and Archana Pai [58], has been selected:

$$
w_{\tilde{r}}(n, m)=t_{s} \sum_{k=-k_{n}}^{k_{n}} \tilde{r}\left[n+\frac{k}{2}\right] \tilde{r}^{*}\left[n-\frac{k}{2}\right] e^{-i 2 \pi m k /(2 N)}
$$

where $k_{n}=\min [2 n, 2 N-1-2 n]$.

This equation, designed for discrete time or space signals, shows a great similarity with the original continuous version, but it also presents some differences and therefore has been referred to as a pseudo-Wigner distribution (PWD). Simply considered, the PWD involves using finite bounds of integration (i.e., a sliding analysis window). This originates a function which, relative to the true $\mathrm{WD}$, is smoothed with respect to the frequency domain only. One important property preserved within the definition given by (B.1) is the inversion property (P9), which is a greatly desirable feature for the recovering of the original signal. Then, local filtering operations are also possible.

Equation (B.1) represents a PWD limited to a spatial interval $[-N / 2, N / 2]$. Here $n$ and $k$ represent the spatial and space-frequency discrete variables, respectively, and $m$ is the shifting parameter, which is also discrete. The values of $n$ represent the sampling positions in the signal considered as a digital replica of an original continuous representation. In this case, the numerical value of the $n^{\text {th }}$ position in the sequence is equal to the value of the analogical signal at position $x=n T$. Here $T$ is recognized as the sampling period and it is the inverse of the sampling frequency. Equation (B.1) originates an $N$-component vector at each position $n$. Furthermore, (B.1) must be interpreted as the discrete Fourier transform (DFT) of product $\widetilde{r}[n, m]=\widetilde{r}[n+m] \widetilde{r}^{*}[n-m]$ in this interval or window.

\section{Data Availability}

The data used to support the findings of this study are included within the article.

\section{Conflicts of Interest}

The authors declare that they have no conflicts of interest.

\section{References}

[1] T. Krawinkel, S. Schn, and A. Bauch, "Application of miniaturized atomic clocks in kinematic GNSS single point positioning," in Proceedings of the 28th European Frequency and Time Forum, EFTF 2014, pp. 97-100, Neuchatel, Switzerland, June 2014.

[2] Y. Jin, B. Luo, X. Hu, and P. Zheng, "A fast positioning method based on high-precision clock for GNSS receivers," in Proceedings of the 2015 IEEE International Conference on Information and Automation, ICIA 2015 - In conjunction with 2015 IEEE International Conference on Automation and Logistics, pp. 809812, Lijiang, China, August 2015.

[3] P. K. Bhatnagar, "Digital Network Synchronization: Basic Concepts," in Engineering Networks for Synchronization, CCS 7, and ISDN:Standards, Protocols, Planning and Testing, pp. 6-41, Wiley-IEEE Press, 1997.

[4] G. L. Weaver, M. Miranian, and J. F. Garstecki, "Composite USO/CSAC timekeeping system," in Proceedings of the 2012 IEEE Aerospace Conference, pp. 1-9, Big Sky, MT, USA, March 2012.

[5] L. A. Mallette, J. White, and P. Rochat, "Space qualified frequency sources (clocks) for current and future GNSS applications," in Proceedings of the IEEE/ION Position, Location and Navigation Symposium, PLANS 2010, pp. 903-908, Indian Wells, CA, USA, May 2010.

[6] H. Hellwig, "Time and frequency applications," IEEE Transactions on Ultrasonics, Ferroelectrics and Frequency Control, vol. 40, no. 5, pp. 538-543, 1993.

[7] INTERNATIONAL STANDARD, “Timing and Synchronization for Time-Sensitive Applications in Bridged Local Area Networks", ISO/IEC/IEEE 8802-1AS:2014(E), IEEE 2011.

[8] R. Miskinis, D. Smirnov, E. Urba, and B. Dzindzelwta, "Timing and synchronization in mobile telecommunication networks," in Proceedings of the 2011 Joint conference of the IEEE International Frequency Control and the European Frequency and Time Forum (FCS), pp. 1-5, San Francisco, CA, USA, May 2011.

[9] J. M. Cohen, H. E. Moses, and A. Rosenblum, "Accurate Time-Keeping and Accurate Navigation," in Proceedings of the 1983 IEEE Military Communications Conference, pp. 729-730, Washington, DC, USA, October 1983.

[10] X. Guo, K. Liu, and J. Yang, "Adaptively precise time synchronization technique for inter-satellite link equipment of navigation satellite," in Proceedings of the 2016 2nd IEEE International Conference on Computer and Communications (ICCC), pp. 1844-1848, Chengdu, China, October 2016.

[11] E. Re, A. Di Cintio, G. Busca, D. Giunta, and M. Sanchez, "Novel Time Synchronization techniques for Deep Space Probes," in 
Proceedings of the 2009 Joint Meeting of the European Frequency and Time Forum (EFTF) and the IEEE International Frequency Control Symposium (FCS), pp. 205-210, Besancon, France, April 2009.

[12] F.-C. Chan, J. Mathieu, and P. Boris, "Stochastic modeling of atomic receiver clock for high integrity GPS navigation," IEEE Transactions on Aerospace and Electronic Systems, vol. 50, no. 3, pp. 1749-1764, 2014.

[13] M. Levesque and D. Tipper, "A Survey of Clock Synchronization Over Packet-Switched Networks," IEEE Communications Surveys \& Tutorials, vol. 18, no. 4, pp. 2926-2947, 2016.

[14] S. Bregni, Synchronization of Digital Telecommunications Networks, John Wiley \& Sons, Ltd, Chichester, UK, 2002.

[15] K. V. Katsaros, W. Chai, N. Wang, G. Pavlou, H. Bontius, and M. Paolone, "Information-centric networking for machine-tomachine data delivery: A case study in smart grid applications," IEEE Network, vol. 28, no. 3, pp. 58-64, 2014.

[16] A. Vallat and D. Schneuwly, "Clock synchronization in telecommunications via PTP (IEEE 1588)," in Proceedings of the IEEE International Frequency Control Symposium Joint with the 21st European Frequency and Time Forum (FCS '07), pp. 334-341, June 2007.

[17] L. Galleani and P. Tavella, "Detection of atomic clock frequency jumps with the Kalman filter," IEEE Transactions on Ultrasonics, Ferroelectrics and Frequency Control, vol. 59, no. 3, pp. 504-509, 2012.

[18] L. Galleani and P. Tavella, "The characterization of clock behavior with the Dynamic Allan Variance," in Proceedings of the IEEE International Frequency Control Sympposium and PDA Exhibition Jointly with the 17th European Frequency and Time Forum, vol. 2003, pp. 5-8, Tampa, Fl, USA, May 2003.

[19] L. Galleani and P. Tavella, "Tracking nonstationarities in clock noises using the dynamic allan variance," in Proceedings of the 2005 IEEE International Frequency Control Symposium and Exposition, pp. 392-396, Vancouver, Canada, 2005.

[20] L. Galleani and P. Tavella, "The dynamic allan variance," IEEE Transactions on Ultrasonics, Ferroelectrics and Frequency Control, vol. 56, no. 3, pp. 450-464, 2009.

[21] I. Sesia, L. Galleani, and P. Tavella, "Application of the dynamic allan variance for the characterization of space clock behavior," IEEE Transactions on Aerospace and Electronic Systems, vol. 47, no. 2, pp. 884-895, 2011.

[22] L. Galleani, "Detection of changes in clock noise using the timefrequency spectrum," Metrologia, vol. 45, no. 6, pp. S143-S153, 2008.

[23] L. Galleani and P. Tavella, "Detection and identification of atomic clock anomalies," Metrologia, vol. 45, no. 6, pp. S127S133, 2008.

[24] L. Galleani, "A Time-Frequency Relationship Between the Langevin Equation and the Harmonic Oscillator," in PseudoDifferential Operators: Groups, Geometry and Applications, pp. 119-131, 2017.

[25] M. G. Amin, D. Borio, Y. D. Zhang, and L. Galleani, "TimeFrequency Analysis for GNSSs: From interference mitigation to system monitoring," IEEE Signal Processing Magazine, vol. 34, no. 5, pp. 85-95, 2017.

[26] Keysight Technologies, "Analyzing Frequency Stability in the Frequency and Time Domains", Application Note, Published in USA, August 11, 2014 5991-4797EN.

[27] D. Allan and J. Barnes, "A Modified "Allan Variance" with Increased Oscillator Characterization Ability," in Proceedings of the Thirty Fifth Annual Frequency Control Symposium, pp. 470475, May 1981.

[28] IEEE Standard Definitions of Physical Quantities for Fundamental Frequency and Time Metrology, IEEE Standard 1139, 1999.

[29] D. W. Allan, "Statistics of atomic frequency standards," Proceedings of the IEEE, vol. 54, no. 2, pp. 221-230, 1966.

[30] J. A. Barnes, A. R. Chi, L. S. Cutler et al., "Characterization of Frequency Stability," IEEE Transactions on Instrumentation and Measurement, vol. IM-20, no. 2, pp. 105-120, 1971.

[31] C. Greenhall, "Does Allan variance determine the spectrum?" in Proceedings of the International Frequency Control Symposium, pp. 358-365, Orlando, FL, USA, 1997.

[32] M. Matejcek and M. Sostronek, "Computation and evaluation allan variance results," in Proceedings of the 2016 New Trends in Signal Processing (NTSP), pp. 1-9, Demanovska dolina, Slovakia, October 2016.

[33] L. Cohen, Time-Frequency Analysis, Prentice Hall, 1994.

[34] E. Wigner, "On the quantum correction for thermodynamic equilibrium," Physical Review A: Atomic, Molecular and Optical Physics, vol. 40, no. 5, pp. 749-759, 1932.

[35] J. Ville, "Theorie et applications de la notion de signal analytique," Cables et Transmission, vol. 2, no. 1, pp. 61-74, 1948.

[36] W. Martin and P. Flandrin, "Wigner-Ville Spectral Analysis of Nonstationary Processes," IEEE Transactions on Signal Processing, vol. 33, no. 6, pp. 1461-1470, 1985.

[37] R. Torres and E. Torres, "Fractional Fourier analysis of random signals and the notion of alpha-stationarity of the Wigner-Ville distribution," IEEE Transactions on Signal Processing, vol. 61, no. 6, pp. 1555-1560, 2013.

[38] J. M. O’Toole, M. Mesbah, and B. Boashash, "Improved discrete definition of quadratic time-frequency distributions," IEEE Transactions on Signal Processing, vol. 58, no. 2, pp. 906-911, 2010.

[39] X. Lv, G. Bi, C. Wan, and M. Xing, "Lvs distribution: principle, implementation, properties, and performance," IEEE Transactions on Signal Processing, vol. 59, no. 8, pp. 3576-3591, 2011.

[40] P. Flandrin and P. Borgnat, "Time-frequency energy distributions meet compressed sensing," IEEE Transactions on Signal Processing, vol. 58, no. 6, pp. 2974-2982, 2010.

[41] T. O. Gulum, P. E. Pace, and R. Cristi, "Extraction of polyphase radar modulation parameters using a Wigner-Ville distribution-Radon transform," in Proceedings of the IEEE International Conference on Acoustics, Speech and Signal Processing (ICASSP '08), pp. 1505-1508, IEEE, Las Vegas, Nev, USA, April 2008.

[42] Y. Wu and D. C. Munson Jr., "Multistatic passive radar imaging using the Smoothed Pseudo Wigner-Ville Distribution," in Proceedings of the IEEE International Conference on Image Processing (ICIP), pp. 604-607, October 2001.

[43] S. Gabarda, G. Cristobal, J. Martínez-Alajarín, and R. RuizMerino, "Detection of anomalous events in biomedical signals by Wigner analysis and instant-wise Rényi entropy," in Proceedings of the 14th European Signal Processing Conference, EUSIPCO 2006, pp. 1-5, September 2006.

[44] F. Akdeniz, I. Kayikiolu, and I. Kaya, "Using Wigner-Ville distribution in ECG arrhythmia detection for telemedicine applications," in Proceedings of the 2016 39th International Conference on Telecommunications and Signal Processing (TSP), pp. 409-412, Vienna, Austria, June 2016. 
[45] F. Akdeniz and T. Kayikcioglu, "Wigner-Ville distribution based ECG arrhythmia detection for telemedicine applications," in Proceedings of the 2016 24th Signal Processing and Communication Application Conference (SIU), pp. 2045-2048, Zonguldak, Turkey, May 2016.

[46] S. C. Pei and J. J. Ding, "Fractional Fourier transform, Wigner distribution, and filter design for stationary and nonstationary random processes," IEEE Transactions on Signal Processing, vol. 58, no. 8, pp. 4079-4092, 2010.

[47] G. F. Boudreaux-Bartels and T. W. Parks, “Time-varying filtering and signal estimation using Wigner distribution synthesis techniques," Institute of Electrical and Electronics Engineers Transactions on Acoustics, Speech and Signal Processing, vol. ASSP-34, no. 3, pp. 442-451, 1986.

[48] L. Yang, L. Zhao, S. Zhou, and G. Bi, "Sparsity-Driven SAR Imaging for Highly Maneuvering Ground Target by the Combination of Time-Frequency Analysis and Parametric Bayesian Learning," IEEE Journal of Selected Topics in Applied Earth Observations and Remote Sensing, vol. 10, no. 4, pp. 1453-1465, 2017.

[49] P. S. Bidari, J. Alirezaie, and J. Tavakkoli, "Shear wave elastography using Wigner-Ville distribution: A simulated multilayer media study," in Proceedings of the 38th Annual International Conference of the IEEE Engineering in Medicine and Biology Society, EMBC 2016, pp. 2873-2876, USA, August 2016.

[50] D. Pang, P. Zhao, and B. Deng, "Cross-terms suppression in Wigner-Ville distribution based on image processing," in Proceedings of the 2010 IEEE International Conference on Information and Automation, ICIA 2010, pp. 2168-2171, China, June 2010.

[51] P. Kartaschoff and H. Brandenberger, Frequency and Time, Burlington, MA: Academic Press, New Jersey, NJ, USA, 1978.

[52] D. W. Allan, N. Ashby, and C. C. Hodge, "The science of timekeeping" Hewlett Packard Application Note 1289, Palo Alto, Santa Clara, Calif, USA, 1997.

[53] P. Tavella, "Statistical and mathematical tools for atomic clocks," Metrologia, vol. 45, no. 6, pp. S183-S192, 2008.

[54] J. L. Semmlow, Biosignal and Biomedical Image Processing MATLAB based Applications, Robert Wood Johnson Medical School New Brunswick, New Jersey, NJ, USA, 2004.

[55] T. A. C. M. Claasen and W. F. G. Mecklenbruker, "The Wigner distributiona tool for time-frequency signal analysis. I. Continuous-time signals," Philips Journal of Research, vol. 35, no. 3, pp. 217-250, 1980.

[56] T. A. C. M. Claasen and W. F. Mecklenbruker, "The Wigner distributiona tool for time-frequency signal analysis Part II. Discrete-time signals," Philips Journal of Research, vol. 35, no. 4/5, pp. 276-300, 1980.

[57] T. A. C. M. Claasen and W. F. G. Mecklenbruker, "The Wigner distribution A tool for time-frequency analysis, Parts I-III," Philips Journal of Research, vol. 35, pp. 276-300, 1980.

[58] E. Chassande-Mottin and A. Pai, "Discrete time and frequency Wigner-Ville distribution: Moyal's formula and aliasing," IEEE Signal Processing Letters, vol. 12, no. 7, pp. 508-511, 2005.

[59] http://www.thefouriertransform.com/pairs/complexGaussian .php.

[60] M. Amirmazlaghani and H. Amindavar, "Modeling and Denoising Wigner-Ville Distribution," in Proceedings of the 2009 IEEE 13th Digital Signal Processing Workshop and 5th IEEE Signal Processing Education Workshop, pp. 530-534, Amirkabir University of Technology, Tehran, Iran, January 2009.
[61] M. Sandsten, "Time-frequency analysis of non-stationary processes-an introduction", Centre for Mathematical Sciences, 2013.

[62] P. Flandrin, R. Baraniuk, and O. Michel, "Time-frequency complexity and information," in Proceedings of the ICASSP '94. IEEE International Conference on Acoustics, Speech and Signal Processing, pp. 329-332, Adelaide, SA, Australia, 1994. 


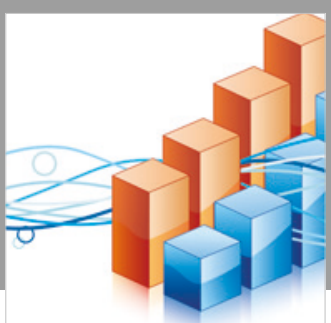

Advances in

Operations Research

\section{-n-m}
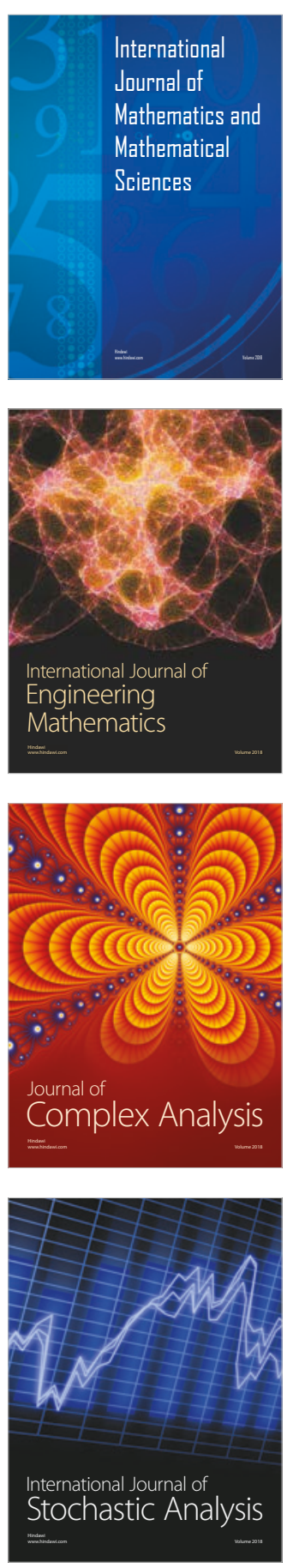
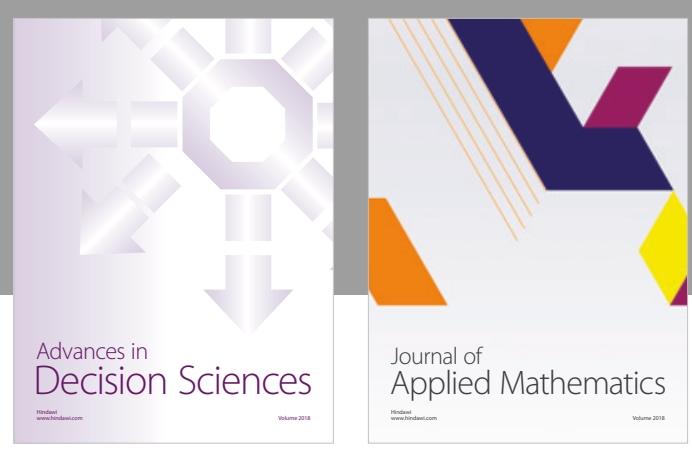

Journal of

Applied Mathematics
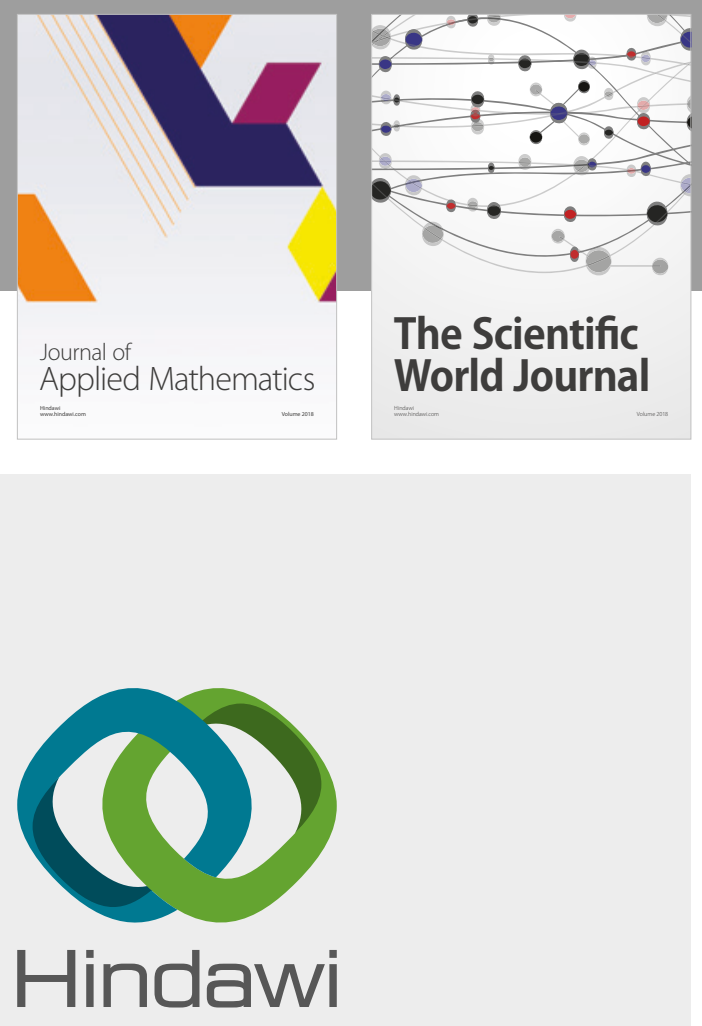

Submit your manuscripts at

www.hindawi.com

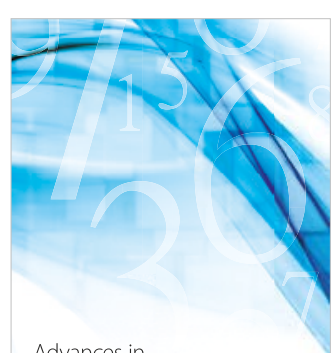

Advances in
Numerical Analysis
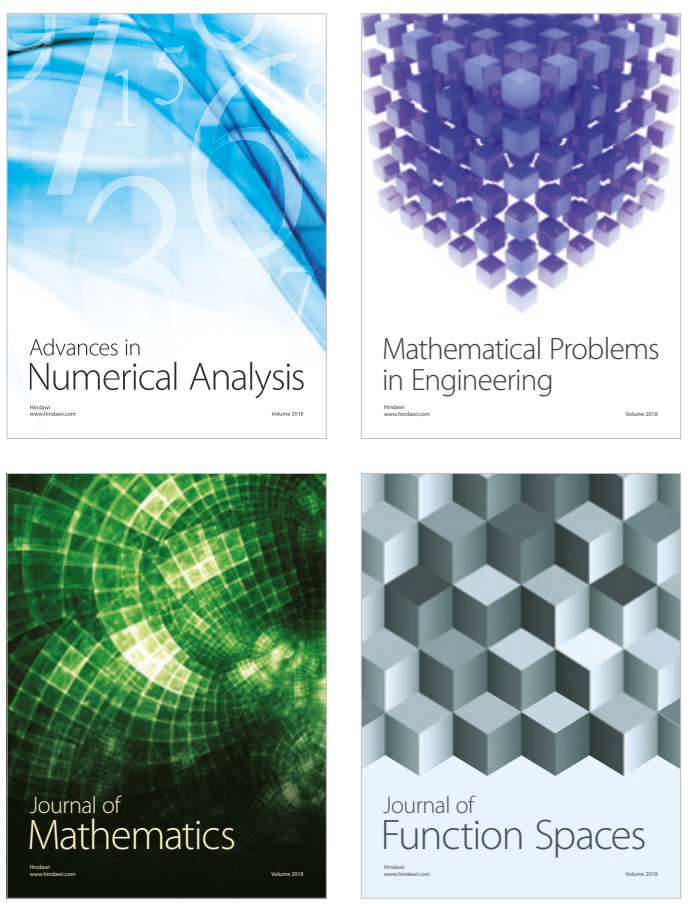

Mathematical Problems in Engineering

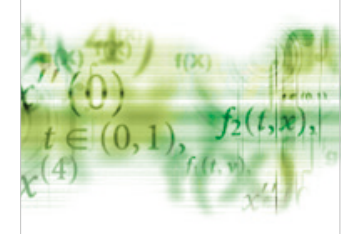

International Journal of

Differential Equations

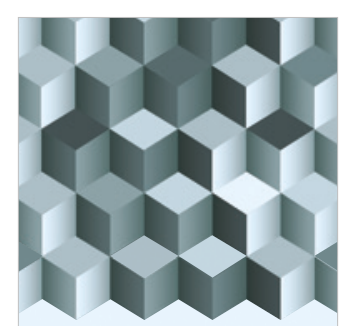

Journal of

Function Spaces

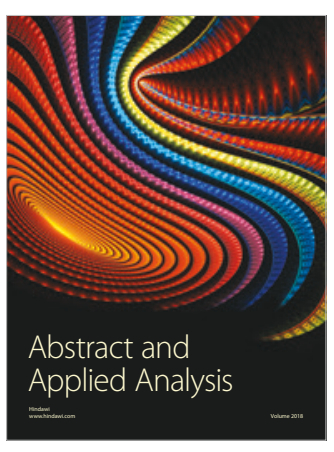

The Scientific

World Journal

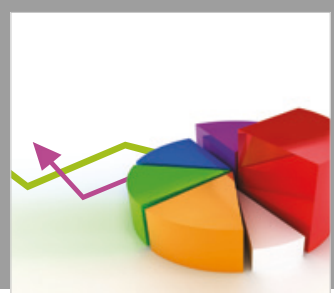

Journal of

Probability and Statistics
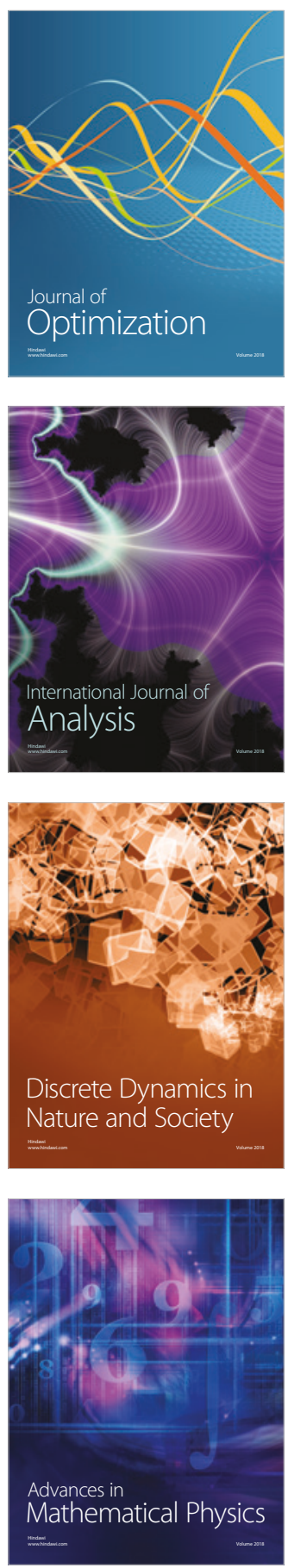